\title{
ILCEA
}

Revue de l'Institut des langues et cultures

d'Europe, Amérique, Afrique, Asie et Australie

33 | 2018

Femmes en résistance du XVIIIe siècle à nos jours

\section{Résistances féministes en faveur de la citoyenneté des femmes}

Feminist Resistance for Women's Citizenship

\section{Bérengère Marques-Pereira}

\section{OpenEdition}

\section{Journals}

Édition électronique

URL : http://journals.openedition.org/ilcea/5310

DOI : 10.4000/ilcea.5310

ISSN : 2101-0609

Éditeur

UGA Éditions/Université Grenoble Alpes

Édition imprimée

ISBN : 978-2-37747-060-0

ISSN : 1639-6073

Référence électronique

Bérengère Marques-Pereira, "Résistances féministes en faveur de la citoyenneté des femmes 》, ILCEA [En ligne], 33 | 2018, mis en ligne le 05 novembre 2018, consulté le 19 avril 2019. URL : http:// journals.openedition.org/ilcea/5310 ; DOI : 10.4000/ilcea.5310

Ce document a été généré automatiquement le 19 avril 2019

(C) ILCEA 


\title{
Résistances féministes en faveur de la citoyenneté des femmes
}

\author{
Feminist Resistance for Women's Citizenship
}

Bérengère Marques-Pereira

\section{Introduction}

1 L'objet de cette contribution est d'aborder quelques modalités majeures des résistances féministes concernant des enjeux fondamentaux liés à la citoyenneté des femmes. La résistance aux tutelles masculines dans une perspective de lutte en faveur des droits civils, sociaux et politiques qui leur étaient ou qui leur sont encore déniés, s'est largement fondée sur la désobéissance à l'ordre des sexes. L'objectif poursuivi par cet article est d'illustrer cette désobéissance par quelques exemples européens (Belgique, France, Grande-Bretagne) et latino-américains (Cône sud) ayant trait au suffragisme et à la politisation du privé et du corporel, des phénomènes essentiels aux revendications des trois vagues du féminisme : le droit de cité pour la première, le droit à la libre disposition de soi pour la deuxième, et le droit à l'autonomie qui se prolongera lors de la troisième vague du féminisme.

2 Mon propos ne vise ni la comparaison entre pays européens et latino-américains, ni l'exhaustivité des revendications de citoyenneté, ce qui exigerait de développer une sociologie historique de politique comparée permettant de cerner les différentes trajectoires nationales et régionales dans l'accès des femmes à l'espace public et politique (Marques-Pereira, 2008, 2015).

Dans la présente contribution, je souhaite mettre en lumière ce que les résistances féministes envers les tutelles masculines qui font obstacle à la citoyenneté des femmes doivent à la désobéissance. Désobéir aux normes de l'ordre des sexes renvoie à la transgression de celles-ci, à leur contournement, au détournement de leur sens androcentré ou patriarcal. Agir en actrices plutôt qu'en victimes de la domination masculine a nécessité la transgression de la norme patriarcale par excellence: la 
séparation entre la sphère publique-politique et la sphère privée-domestique. C'est en exerçant les libertés d'association et de réunion, quand bien même celles-ci leur étaient interdites ou déniées, que les femmes ont accédé à l'espace public pour revendiquer leurs droits de citoyennes.

Le contournement des normes patriarcales s'est aussi fondé sur la construction d'un espace public autonome par rapport à l'État, aux partis politiques ou aux organisations internationales. Cette construction tend à se défaire de la normativité juridico-politique qui enserre les revendications féministes dans le moule du Droit et des droits, en maintenant une indépendance par rapport aux financements et aux agendas politiques nationaux et internationaux. L'enjeu est plutôt de produire une identité politique fé ministe autonome.

Détourner les normes de leur sens patriarcal c'est notamment se baser sur le mécanisme de retournement du stigmate. Ainsi, les rôles assignés ou attendus de mères, loin de se réduire à un maternalisme prescrit et naturalisé, peuvent se transformer, dans certaines conjonctures politiques, en un maternalisme souscrit et revendiqué pour obtenir des droits de citoyenneté. En effet, si le maternalisme peut être compris à la fois comme une représentation et une pratique qui réduit l'identité des femmes à celle de mère, il faut noter que la revendication des droits de citoyenneté s'est appuyée, en Europe et en Amérique latine, sur une dialectique de l'égalité et de la différence modifiant les rôles naturalisés de mère en une fonction sociale à valoriser politiquement et socialement.

Ces dynamiques de désobéissance sont la manifestation de deux processus conjoints: celui de la subjectivation et celui de l'individuation des femmes, processus qui se trouvent au cœur de l'acquisition et de l'exercice de leurs droits de citoyenneté. De tels processus ont été de pair avec une transnationalisation du féminisme, que ce soit dans le cadre des dynamiques institutionnelles ou dans celui de dynamiques protestataires d'engagement.

7 Aussi, ma démarche comporte-t-elle deux versants: d'une part, la subjectivation et l'individuation des femmes que comportent leurs résistances vues sous l'angle de la désob éissance; d'autre part, les dynamiques institutionnelles et protestataires qui se développent à l'aune de la transnationalisation du féminisme, moteur de cette subjectivation et individuation. Une telle démarche se fonde, au plan méthodologique, sur la sélection de moments chronologiques les plus saillants dans les revendications et les acquis de la politique féministe de la citoyenneté (droit de suffrage, droit à la libre disposition de soi, droit à l'autonomie). Cette démarche s'inscrit dans une problématique sociologique qui refuse l'opposition traditionnelle entre contestation et institutionnalisation mise en œuvre par la sociologie des mouvements sociaux et la sociologie des politiques publiques. Notre perspective socio-historique et politique s'ancre dans le parcours du réseau francophone et pluri-disciplinaire État et rapports sociaux de sexes (Del Re, Gautier, Heinen, Jenson, Marques-Pereira \& Spensky, 2013).

\section{Subjectivation et individuation au regard de la citoyenneté des femmes}

8 Transgresser, contourner ou détourner les normes de l'ordre des sexes sont l'expression de deux processus conjoints portés par le féminisme : la subjectivation et l'individuation des femmes. Le féminisme ne se réduit pas à l'obtention de l'égalité des droits. Certes, il s'agit d'un axe central de luttes, puisque l'acquisition de ces droits a permis une 
dynamique d'individuation des femmes (en rupture avec l'essentialisme qui renvoie à un être abstrait, la femme, plutôt qu'à des êtres concrets, les femmes, actrices et agentes de la matérialité des rapports sociaux dans lesquelles elles s'insèrent). Mais le féminisme contemporain est également porteur d'une autre facette de luttes : celle de la liberté, celle de la libération des femmes, celle de l'indépendance, celle de l'autonomie. Le féminisme comme processus de subjectivation se traduit ainsi par une dialectique de l'égalité et de la liberté, qui se matérialise à travers la notion d'autonomie organisationnelle, politique et personnelle (Lamoureux, 2016).

9 L'exercice de la citoyenneté se fonde sur la capacité de la personne à agir en tant que sujet autonome qui n'a plus à se référer aux seules identités prescrites pour exister socialement et politiquement, mais devient capable de revendiquer aussi des identités souscrites. La pratique de la citoyenneté va dès lors de pair avec la capacité des individus à reconnaître leurs problèmes à la fois comme singuliers et collectifs au regard des processus de construction de leur autonomie privée et publique. Cela suppose l'accès à des ressources permettant aux individus de rechercher des solutions à leurs problèmes à travers une participation sociale et politique, qu'elle soit d'ordre contestataire, institutionnel, local, international, associatif ou partisan.

La citoyenneté repose ainsi sur une pratique qui légitime le "droit à avoir des droits ", selon la belle formule d'Hannah Arendt, c'est-à-dire le droit de revendiquer des nouveaux droits et le droit d'exercer les droits existants. Le processus de subjectivation s'appuie sur des pratiques d'engagement et de résistance qui permirent au féminisme d'être un moteur majeur débouchant sur l'acquisition des droits de citoyennes.

11 D’emblée le féminisme se conçoit comme un mouvement international (Klejman, 1989). Le féminisme émerge également en relation avec une conception des droits humains universels issus de la Révolution américaine et de la Révolution française et se construit à partir d'une contradiction : l'affirmation de principes universels d'égalité face à la réalité des discriminations qui font des femmes des citoyennes de seconde classe. Au-delà des luttes menées à l'encontre de ces discriminations, le féminisme se fonde sur la reconnaissance de l'oppression des femmes et de la domination masculine à leur égard. Cette reconnaissance renvoie au caractère social et systémique des relations stratégiques de pouvoir des hommes sur les femmes et, par conséquent, sur la possibilité de leur transformation.

12 Tant en Europe qu'en Amérique latine, le féminisme est ce mouvement collectif, fait de luttes de femmes, et parfois d'hommes, qui naît dès la deuxième moitié du XIX ${ }^{e}$ siècle et se prolonge dans la première moitié $\mathrm{du} \mathrm{xx}^{\mathrm{e}}$ siècle, en revendiquant pour les femmes le droit à l'éducation et à l'instruction, l'accès à la culture et au savoir, l'insertion dans le monde du travail professionnel, dans le mutuellisme et dans le syndicalisme, le droit à l'égalité civile et politique avec les hommes. De telles revendications expriment ce qui est au cœur de la citoyenneté : l'individuation. Cette dynamique d'individuation des femmes repose sur l'accès au savoir, l'accès à l'échange monétaire - la monnaie comme moyen de paiement permettant à l'individu de se libérer de toute dette économique et sociale -, l' accès à l'échange contractuel - le contrat juridique dont les formes de l'égalité et de la liberté définissent l'individu comme citoyen libéré des assujettissements tutélaires - et l' accès à l'échange délibératif - la délibération politique comme capacité de l'individu d'exister dans l'espace public comme sujet politique - (Marques-Pereira, 2005). 
13 La deuxième vague du féminisme émerge au milieu des années 1960 et au début des années 1970. Elle est également marquée par une dynamique d'individuation des femmes, qui élargit leur revendication de disposer librement d'elles-mêmes à une politisation du privé (par exemple la dénonciation de l'assignation des femmes au travail domestique gratuit, ou une socialisation du travail domestique) et à une politisation du corporel (lutte en faveur des droits reproductifs et sexuels et contre la violence de genre que ce soit dans l'espace privé ou dans l'espace public). Ces même processus ont lieu également en Amérique latine. Mais les mouvements de femmes latino-américaines, en particulier ceux du Cône sud, ont connu une trajectoire tridimensionnelle. Outre un mouvement populaire qui a transformé des stratégies de survie économique en revendications socio-politiques, les mouvements de femmes se sont également constitués autour de revendications féministes, d'une part, et autour de protestations contre les violations des droits humains, d'autre part.

14 La troisième vague du féminisme se développe à partir des années 1980-1990, prolongeant la revendication de l'autonomie organisationnelle, politique et personnelle dans une perspective intersectionnelle et dé-coloniale qui, à l'aune de la globalisation, met en cause les cadres stato-nationaux de la citoyenneté et permet de reconnaître la diversité des oppressions de genre, de classe et de race ainsi que le pluralisme des positions féministes (Cirstocea \& Giraud, 2016). En Europe, une telle optique donne lieu à des revendications d'affirmation identitaire notamment autour de la question du voile. En Amérique latine, elle met en avant des revendications identitaires et de distribution d'afro-descendantes et d'indigènes.

15 L'accès au droit de cité et la politisation du privé et du corporel se sont appuyés sur la transgression de la norme de la séparation entre le privé et le public. C'est ce que firent les femmes luttant pour le suffragisme, pour qui il fallait accéder à l'espace public en exer çant les libertés d'association et de réunion et en utilisant des répertoires d'actions collectives d'ordre institutionnel et protestataire. En effet, les suffragettes britanniques firent preuve de désobéissance civile et furent considérées comme des criminelles en raison de leur infraction à la loi, infraction qui représentait un acte d'affirmation de leur appartenance à la communauté politique. Faisant physiquement barrage de leur corps, les suffragettes désobéissaient en outre à la norme sociale, ce qui permettait de les qualifier d'hystériques et, ce faisant, de disqualifier leur engagement politique (BentouhamiMolino, 2015). Que ce soit en Europe ou en Amérique latine, la revendication féministe du droit de suffrage s'est appuyée sur l'usage d'une stratégie rhétorique de l'égalité et de la différence, manifestant ainsi un détournement de la norme patriarcale du maternalisme assigné en un maternalisme souscrit.

La politisation du privé dénonçant l'assignation des femmes à l'espace domestique et au travail domestique gratuit représente une résistance à la fois privée et publique : ce qui était vécu sur le mode de l'évidence sociale et du cela va de soi, inscrits dans les rôles prescrits des femmes, débouche sur une mise en cause de la naturalisation des hiérarchies, des places et des fonctions inhérentes à l'ordre des sexes. Politiser le privé a $\mathrm{pu}$, par exemple, prendre la forme de cuisines populaires en Amérique latine. Si certaines d'entre elles n'ont pu maintenir leur caractère autogestionnaire d'origine tant le clientélisme d'État a contribué à fractionner le féminisme dans certains pays (Rousseau, 2009), il n'en demeure pas moins que les cuisines qui ont réussi à sauvegarder leur autonomie, ont représenté un détournement de la norme de séparation entre le privé et le public. À mi-chemin entre le privé et le public, l'auto-organisation collective de la 
pauvreté a permis à certaines femmes de vivre un maternalisme souscrit plutôt que prescrit, de faire l'apprentissage d'un sens civique local, d'accéder au statut de leaders de leur communauté locale et de développer des réseaux de solidarité.

Autre exemple célèbre de transgression et de détournement de la norme de séparation entre le privé et le public: les Mères de la Place de Mai. À partir de la projection dans l'espace public de leurs rôles privés, traditionnels, de mères, d'épouses, de compagnes, les Mères de la Place de Mai défient ouvertement les militaires argentins, leur demandant des comptes sur la disparition de leurs proches. Ce n'est pas le seul exemple en Amérique latine où des femmes affrontent publiquement un espace dangereux en butte à la violence de la dictature militaire, transformant ainsi un maternalisme prescrit en maternalisme souscrit, mais il nous a semblé particulièrement emblématique.

La politisation du corporel représente aussi une résistance à la fois privée et publique. Les luttes des femmes contre les violences masculines à leur égard sont essentielles à leur estime de soi en tant que personne. Phénomène à la fois individuel et collectif, l'estime de soi ne se construit sans doute pas de la même manière dans les sociétés individualistes et dans les sociétés qui portent la marque holiste du populisme et du clientélisme. Mais dans un cas comme dans l'autre, l'estime de soi demeure fondamentale à l'exercice d'une affirmation d'identité politique sans laquelle il ne peut y avoir de revendication portant sur « le droit à avoir des droits ». Ainsi, la violence de genre (construite sur le continuum du harcèlement de rue au féminicide en passant par la violence domestique, le viol dans l'espace privé et public ainsi que le viol comme arme de guerre) constitue-t-elle un rappel à l'ordre des sexes et un véritable déni de la citoyenneté des femmes, d'autant que l'accès à la justice est tellement réduit que l'on peut considérer que l'état n'exerce pas son monopole de la violence légitime et sa fonction de garant de la sûreté personnelle lorsqu' il s'agit des femmes. Rien d'étonnant dès lors que la lutte contre cette violence soit un enjeu international, d'autant plus saillant en Amérique latine que celle-ci constitue la région du monde où les taux de violences à l'égard des femmes, sont les plus élevés (Friés \& Hurtado, 2010).

Par ailleurs, la lutte en faveur du droit d'accès à l'avortement dans de bonnes conditions sanitaires et psychologiques a consisté à transgresser le tabou qui pesait sur sa pratique. Faire de ce qui était éminemment intime un enjeu public, a requis de briser la loi du silence qui entourait sa pratique. Tel fut le sens des manifestes de femmes belges et franç aises proclamant publiquement avoir eu recours à un avortement, transgression ouverte de la loi pénale. En effet, la désobéissance civile fut nécessaire dans des pays comme la Belgique et la France face à des lois éminemment patriarcales dans lesquels l'interdit pé nal en la matière est conçu comme une protection de l'ordre public et des familles. Ce recours à la désobéissance civile était l'expression d'une résistance publique, non clandestine, de celles qui avaient eu recours à un avortement ainsi que des médecins et de membres du personnel para-médical qui pratiquaient l'avortement médical dans de bonnes conditions sanitaires et psychologiques (Marques-Pereira, 2017).

Si, en Amérique latine, un pays tel que l'Uruguay ou la ville de Mexico ont dépénalisé partiellement l'avortement (Correa \& Pecheny, 2016) et constituent une exception à la $r$ ègle de l'interdit pénal, le poids des positions de la hiérarchie ecclésiastique se fait toujours sentir, même dans des pays gouvernés au centre-gauche, tel le Chili de Michelle Bachelet (Le Trividic, 2013 ; Miranda-Pérez \& Gómez-Medina, 2014) ou dans des pays qui ont été gouvernés par des femmes, telle l'Argentine de Cristina Kirschner (Felitti, 2014) ou le Brésil de Dilma Rousseff qui a dû renoncer à ses positions en faveur du droit à 
l'avortement pour être élue à la présidence du pays. Notons toutefois qu'un pays comme le Chili connaît des pratiques de désobéissance civile en faveur de ce droit. En effet, des féministes lesbiennes ont mis sur pied un centre d'appel téléphonique diffusant une information portant sur les possibilités d'avortement médicamenteux à la maison, transgressant ainsi la loi pénale et contournant un pouvoir médical dont les positions anti-choix sont hégémoniques; suite au dépôt d'un projet de loi dépénalisant l'avortement thérapeutique à la Chambre le 17 mars 2016, ces féministes ont fermé ce centre d'appel et ont diffusé un manuel d'information (López Escriva, 2015).

En Europe, l'activisme pro-choix a pu montrer toute son efficacité et sa vigilance face aux tentatives de régressions législatives, comme en Espagne, et même dans le cas de lois déjà restrictives comme en Pologne (Heinen, 2013). Une avancée du droit à l'avortement a é mergé en France et au Luxembourg qui ont supprimé la notion d'état de détresse requis comme condition pour permettre l'interruption volontaire de grossesse pour la femme enceinte. La vigilance pro-choix s'exerce également à l'égard de l'activisme discursif du Vatican et de ses relais, notamment au Parlement européen.

De manière générale, transgresser les normes, les contourner, les détourner de leur sens patriarcal, sont autant d'expressions d'une résistance des femmes lors de leurs engagements politiques féministes (au sens large du terme). Autant d'éléments qui furent cruciaux au regard de ces qualités démocratiques que sont la liberté (au sens de libération des tutelles masculines) et l'égalité (au sens de rupture avec les formes de domination paternalistes et de dépendance personnelle aux hommes). Autant d'éléments dont l'un des moteurs majeur fut le féminisme.

\section{La transnationalisation du féminisme : dynamiques institutionnelles et protestataires}

Dès le $\mathrm{XIX}^{\mathrm{e}}$ siècle, le féminisme fut construit au plan international, à travers une autonomie organisationnelle mise sur pied, notamment, au moyen d'associations féministes internationales et de congrès féministes internationaux. De 1880 à 1930, la mobilisation internationale ayant trait à la paix, au suffrage des femmes, à l'accès à l'éducation, à l'égalité des salaires et au bien-être social, transcende les frontières nationales.

$\mathrm{Au}$ lendemain de la seconde guerre mondiale et dès la création de l'ONU, le féminisme se transnationalise. En effet, il s'engage dans des relations transnationales, quand il entre en relation avec des mouvements sociaux ou des acteurs non institutionnels, ou à travers des frontières nationales, avec un autre État ou une institution internationale ou supranationale.

Entre les deux premières vagues du féminisme, plusieurs organisations de femmes ont continué de lutter pour l'exercice de leurs droits de citoyenneté et ces luttes ont abouti, au lendemain de la Seconde Guerre mondiale, à la mise sur pied au sein des Nations unies de la Commission de la condition de la femme (1946). Cette Commission a œuvré à l'établissement de plusieurs conventions fondées sur l'égalité entre femmes et hommes, notamment la Convention sur les droits politiques des femmes (1954), la Convention sur la nationalité des femmes mariées (1957), la Convention sur le consentement au mariage et sur l'âge minimum (1962) et la Convention sur l'élimination de toutes les discriminations à l'égard des femmes - CEDEF, 1979 - (Rozée, 2011). 
Tant en Amérique latine qu'en Europe, la reconnaissance de l'égalité entre femmes et hommes s'appuie aujourd'hui sur ces conventions.

L'élaboration d'une nouvelle normativité sociale à travers les conventions internationales (rendues contraignantes pour les législations nationales, dès lors que ces conventions sont ratifiées par les parlements nationaux) a été concomitante à la construction d'un espace international de la cause des femmes ${ }^{1}$. En outre, la Commission onusienne de la condition de la femme est à l'origine de l'organisation de conférences internationales ayant trait aux droits des femmes, la première ayant eu lieu à Mexico en 1975 et la dernière à Beijing en 1995. Dès 1985, une troisième vague du féminisme transnational é merge à la faveur de la Conférence mondiale des droits des femmes, organisée à Nairobi par les Nations unies. Dès ce moment, les ONG du Sud commencent à défier la domination idéologique des ONG du Nord dans le cadrage de l'agenda international des droits des femmes. Des ponts se construisent petit à petit entre les perspectives féministes du Nord axées sur les droits reproductifs et sexuels et les perspectives féministes du Sud centrées sur le développement de la lutte contre la pauvreté. Des enjeux tels que la lutte contre la violence de genre et la reconnaissance des droits des femmes en tant que droits humains constituent de tels ponts et c'est dans ce sillage que se développent des réseaux féministes transnationaux.

8 En Amérique latine et en Europe, les associations de femmes ont acquis, à partir des années 1990, une dimension institutionnelle. Celle-ci se traduit notamment par leur participation aux processus préparatoires des conférences mondiales et a impulsé à cette occasion, une transnationalisation des mouvements de femmes qui s'exprime à travers des dynamiques à la fois institutionnelles et protestataires.

La transnationalisation des discours et des pratiques de ces mouvements a permis aux femmes de légitimer leurs revendications et de faire pression sur les partis politiques et les gouvernements pour inscrire celles-ci à l'agenda politique (Keck \& Sikking, 1998). Ce processus a aussi favorisé la création de réseaux tels que le Lobby européen des Femmes ${ }^{2}$ ou la création de réseaux thématiques transnationaux sur des enjeux tels que la violence à l'encontre des femmes, les droits reproductifs et sexuels, la parité, etc. ${ }^{3}$. Ce processus a, en outre, débouché sur une incorporation sélective des revendications féministes en même temps que sur une dynamique de re-signification de ces dernières par les gouvernements (Alvarez, 2004). Cette neutralisation des revendications n'empêche pas qu'au niveau national ou régional, les suivis quinquenaux de la conférence de Beijing, en 2000 et jusqu'à ce jour, ait donné lieu de la part des associations de femmes à une évaluation des engagements accomplis par les gouvernements des différents pays, gouvernements qui ont dû, par ailleurs, en rendre compte devant leurs parlements.

En effet, grâce à la vigilance exercée par les associations et ONG féministes, les engagements pris par les États lors de ces conférences ont notamment abouti à la mise en place d'agences étatiques et de plans d'action concernant l'égalité entre hommes et femmes, que ce soit en Europe ou en Amérique latine.

De manière générale, l'élaboration d'une nouvelle normativité sociale en matière de droits des femmes s'est concrétisée par la recommandation onusienne en faveur de l'institutionnalisation du gendermainstreaming dans les différentes politiques publiques. Le gendermainstreaming demande aux États d'évaluer ex-ante et ex-post les effets différenci és, pour les femmes et pour les hommes, de la mise en œuvre des politiques publiques. 
Dans cette perspective, les femmes ne seraient plus considérées comme une catégorie à part, et les politiques d'égalité de genre un secteur à part.

De manière concomitante, la dynamique d'institutionnalisation de l'espace international de la cause des femmes s'est traduite par la création, par l'Assemblée générale des Nations unies en juillet 2010, d'une nouvelle instance onusienne pour l'égalité des sexes et l'autonomisation des femmes : l'ONU Femmes. Son rôle est d'apporter un appui aux organes intergouvernementaux dans l'élaboration des politiques, des règles et des normes mondiales. L'ONU Femmes a également vocation à assurer la coordination et le suivi au niveau du système onusien de ses propres engagements en faveur de l'égalité des sexes.

33 La transnationalisation des discours, des pratiques et des enjeux féministes ne s'est pas seulement appuyée sur ce versant institutionnel. Un versant protestataire s'est également développé.

34 Au Sud comme au Nord, les mouvements de femmes de la deuxième vague ont été rapidement confrontés à un enjeu politique majeur : la participation aux institutions publiques. Si en général, l'appel à la participation aux institutions étatiques et à la d émocratie libérale est accueilli favorablement tant par les féministes libérales et les féministes latino-américaines dites institutionnelles que par celles issues de la gauche partisane et syndicale, il faut rappeler que, dès le début de cette deuxième vague, des féministes dites autonomes en Amérique latine ou dites révolutionnaires en Europe occidentale, remettent en question cette entrée dans les institutions et en politique institutionnalisée. Mais deux décennies plus tard, les luttes en faveur de la parité tout comme la légitimation, par les Nations unies, de cette revendication et des acteurs qui la portent, ont largement étouffé cette composante des mouvements féministes.

Un exemple important de cette composante en Amérique latine réside dans la tenue, depuis 1981, des encuentros feministos (Olea Mauleón, 1998). Ces rencontres féministes transnationales latino-américaines en appellent à l'autonomie des mouvements de femmes et sont un espace public où peut se construire une identité féministe autonome. Leur enjeu n'est pas de participer à l'élaboration de nouvelles normes juridiques, mais plutôt de mettre en cause la réduction institutionnelle des revendications féministes au moule du Droit et des droits. Ce que les féministes dites autonomes reprochent aux féministes dites institutionnelles, c'est d'avoir détourné la praxis de subversion du féminisme au profit d'une logique de la subvention et d'une logique technicobureaucratique.

36 Par ailleurs, ces encuentros ont été un lieu de confrontation à la diversité ethnique et sexuelle (García \& Valdivieso, 2006). Ainsi, ce que les mouvements de femmes afrodescendantes et autochtones reprochent aux féministes institutionnelles et autonomes est le fait d'occulter le caractère intersectionnel des enjeux et des situations que vivent les femmes pauvres racisées (Falquet, 2011).

37 À telle enseigne qu'elles ont mis sur pied, depuis 1995, de nouveaux encuentros transnationaux qui permettent la mise en avant de deux éléments majeurs conjoints : $d$ 'une part, la reconnaissance du sexisme comme un élément interne aux communautés et organisations indigènes et afro-descendantes; d'autre part, la revendication d'une autonomie des espaces organisationnels pour les femmes en butte à la pauvreté et au racisme. 

dernier entretient avec le mouvement altermondialiste, en particulier à l'occasion de la tenue des forums sociaux ou encore à l'occasion des actions entreprises par la Marche mondiale des femmes (MMF). Mise sur pied à Québec lors du Sommet des Amériques, la MMF se développe depuis lors dans différents continents, dont l'Europe et l'Amérique latine (Dufour \& Giraud, 2010; Giraud, 2016). La cible de la MMF est moins les États et les organisations internationales que d'autres mouvements sociaux, visant ainsi la construction d'une politique de coalition anti-néolibérale et féministe. Dans cette perspective, la production identitaire féministe à travers un entre soi non mixte poursuit l'objectif d'une mixité égalitaire au sein des différents mouvements sociaux antinéolibéraux.

exemples de transnationalisation "par le bas» (Alvarez, Faria \& Nobre, 2004) nous mettent en présence d'une résistance aux normes patriarcales par la construction d'espaces publics autonomes ainsi qu'à travers des marches ou des rencontres d'ordre protestataire.

en faveur de l'égalité des sexes, il n'en demeure pas moins que leur préoccupation de déconstruction des systèmes d'oppression patriarcale et de domination masculine est un élément majeur dans les représentations collectives qui mettent en cause l'ordre des sexes. À ce titre, la citoyenneté des femmes leur est redevable d'une pensée libertaire qui rappelle ce que la recomposition des rapports sociaux vers l'égalité de genre doit à la contestation des systèmes d'oppression et de domination.

41 Enfin, la transnationalisation du féminisme "par le bas » nous met en présence d'un $\mathrm{f}$ éminisme dé-colonial et intersectionnel. Celui-ci met en cause le féminisme institutionnel hégémonique lorsque celui-ci s'arroge, par exemple, les prérogatives de la lutte contre les violences faites aux femmes du Tiers Monde, sans analyser les composantes classistes et racistes à l'œuvre dans les phénomènes conjoints de l'exploitation et de la domination, en particulier à l'heure de la globalisation (Verschuur \& Destremau, 2012).

\section{Conclusion}

Que peut-on conclure de ces formes de résistance qu'ont été les engagements des femmes en faveur d'une politique de citoyenneté ? La cause des droits des femmes s'est adossée à des valeurs, des projets de lutte, des expériences historiques et contemporaines.

Les valeurs d'égalité et de liberté : elles sont construites historiquement sur l'autonomie par rapport aux tutelles masculines, qu'elles soient paternelles, maritales ou fraternelles. Autonomie qui suppose le droit à l'égalité de tous les individus et l'égalité des droits entre femmes et hommes dans les différents domaines du Droit. Autonomie qui s'ancre dans la construction d'une indépendance organisationnelle et personnelle.

Le projet de lutte contre les discriminations : il s'agit de s'opposer à la transformation des différences en inégalités sociales. Mais il y a plus. Il y a aussi une lutte d'affirmation identitaire: acter d'avoir été exclues comme femmes et revendiquer l'inclusion économique, sociale, politique et culturelle en tant que femmes. Il y va de l'estime de soi au féminin, c'est-à-dire du refus de la norme masculine comme norme de référence et de révérence, refus qui traduit des dynamiques de retournement du stigmate et une dénonciation des systèmes d'oppression des femmes. Refus, pour le dire succinctement, 
de l'universalisation du masculin. En outre, l'estime de soi passe, pour les femmes pauvres racisées, par la mise en cause d'un féminisme hégémonique aveugle aux discriminations entre femmes, discriminations qui puisent leur ancrage dans les rapports sociaux de classe et de race.

Les expériences historiques et contemporaines : celles de la conquête du suffrage féminin, des luttes en faveur de l'égalité des salaires, en faveur du droit à l'avortement et à la contraception, des réformes dans le droit de la famille, etc. Ou, plus proche de nous : les mobilisations menées contre la régression de certains acquis, tels que le droit à l'avortement, ou la politisation de nouveaux enjeux tels que les violences de genre (viol, violences sexuelles à l'encontre des femmes, prostitution, etc.).

Toutes ces luttes passent par des pratiques sociales et politiques qui sont à la fois d'intégration et d'autonomie: intégration dans les différentes institutions de l'espace public; autonomie des associations et des groupes qui ont politisé les enjeux de genre. Cette dialectique de l'intégration et de l'autonomie ne s'est pas déroulée sans conflits. Mais quels que soient ces conflits, tous sont porteurs des résistances inhérentes aux engagements politiques au sens large du terme.

\section{BIBLIOGRAPHIE}

Alvarez Sonia (2004), « Advocating Feminism. The Latin American Feminist NGO Boom », L. Ricciutelli, A. Miles \& N. Mcfadden (dir.), Feminist Politics Activism and Vision, Londres : Zed Books, 122-148.

AlVAREZ Sonia, FARIA Nalu \& NOBRE Miriam (2004), « Another (also feminist) world is possible. Constructing transnational spaces and global alternatives from the movements ", J. Sen, A. Anand, A. Escobar \& P. Waterman (dir.), World Social Forum: Challenging Empires, Delhi : Viveka Foundation, 199-206.

Bentouhami-Molino Hourya (2015), Le dépôt des armes. Non-violence et désobéissance civile, Paris : PUF.

BERENI Laure (2015), La bataille de la parité. Mobilisations pour la féminisation du pouvoir, Paris : Economica.

CIRSTOCEA Ionna \& GIRAUD Isabelle, «Pluralisme dans les mouvements féministes contemporains » , I. Cirstocea \& I. Giraud (dir.), Pluralisme dans les mouvements féministes contemporains, Paris : L'Harmattan, 29-49.

CORREA Sonia \& PECHENY Mario (2016), Abortus Interruptus. Politica y reforma legal del aborto en Uruguay, Montevideo : International Women's Health Coalition.

Del Re Alisa, Gautier Arlette, Heinen Jacqueline, Jenson Jane, MARques-Pereira Bérengère \& S PENSKY Martine (2013), «Quelle citoyenneté pour les femmes ? État des lieux et perspectives (1987-2012). Groupe État et rapports sociaux de sexes », Cahiers du Genre, 54, 67-92.

DUFOUR Pascale \& GIRAUD Isabelle (2010), Dix ans de solidarité planétaire. Perspectives sociologiques sur la Marche mondiale des femmes, Montréal : Les éditions remue-ménage. 
FALQUET Jules (2011), « Les féministes autonomes latino-américaines et caribéennes : vingt ans de critique de la coopération au développement », Recherches féministes, 2, 39-58.

FeLITTI Karina (2014), « L'avortement en Argentine : politique, religion et droits humains », A. Gautier \& C. Grenier-Torres (dir.), Les droits reproductifs 20 ans après Le Caire, Autrepart, 70, 73-90.

FRIÉS Lorena \& HURTADO Victoria, « Estudio de la informacion sobre la violencia contra la mujer en América Latina y el Caribe », Mujer y desarrollo, 99, 1-57.

GARCIA Carmen \& VALDivieso Magdalena (2006), « Una aproximación al movimiento de mujeres en América Latina », Observatório Social de América Latina, 18, 41-56.

GIRAUD Isabelle (2016), « Intégrer la diversité des oppressions dans la Marche mondiale des femmes ", I. Cirstocea \& I. Giraud (dir.), Pluralisme dans les mouvements féministes contemporains, Paris : L'Harmattan, 95-112.

HEINEN Jacqueline (2013), « Le droit de choisir en question. Hier et aujourd'hui », D. Paternotte \& N. Nagels (dir.), Imaginer la citoyenneté, Louvain-la-Neuve : Academia-L'Harmattan, 59-77.

KeCK Margaret \& SIKKING Katherin (1998), Activist beyond Borders, Ithaca : Cornell University Press, 165-198.

KLEJMAN Laurence (1989), « Les Congrès féministes internationaux », Mil neuf cent, 7, 71-86.

LAMOUReuX Diane (2016), Les possibles du féminisme. Agir sans «nous », Montréal : Les éditions remue-ménage.

LE TRIVIDic HARRACHe Lila (2013), Démocratie et sexualité. Politisation de la pilule du lendemain dans le Chili de la Concertación (1990-2010), Paris : IHEAL.

LóPEz EsCRIVA Fernando (2015), The Abortion Hotline, en ligne sur <https://vimeo.com/146408056> (17 décembre 2016).

MARQUES-PEREIRA Bérengère (2005), « Approche de la citoyenneté des femmes comme processus d'individuation », B. Marques-Pereira \& P. Meier (dir.), Genre et Politique en Belgique et en francophonie, Bruxelles : Academia-Bruylant, 105-113.

MARQUeS-PEREIRA Bérengère (2008), « L'accès des femmes à l'espace public : du local au national, de l'international au transnational. L'exercice de la responsabilité publique et les rapports de genre en Amérique latine », Nuevo Mundo, Mundos Nuevos, <http:// nuevomundo.revues.org//34293>.

MARQUES-PEREIRA Bérengère (2010), « Le féminisme latino-américain au niveau transnational », B. Marques-Pereira, P. Meier \& D. Paternotte (dir.), Au-delà et en deça de l'État. Le genre entre dynamiques transnationales et multi-niveaux, Louvain-la-Neuve : Academia-Bruylant, 79-92.

MARQUES-PEREIRA Bérengère (2015), « Quelques jalons pour une politique comparée de la citoyenneté des femmes en Amérique latine », K. Bergès, D. Burgos-Vigna, M. Yusta Rodrigo \& $\mathrm{N}$. Leduc (dir.), Résistantes, militantes, citoyennes. L'engagement politique des femmes au XX et XXI siècles, Rennes : Presses universitaires de Rennes, 19-31.

MARQUES-PEREIRA Bérengère (2017), "Le droit à l'avortement : une mobilisation persistante», C. Gobin \& D. Paternotte (dir.), Contester en Belgique : raisons, cadres et mobilisations, Louvain-laNeuve : Academia-L'Harmattan, à paraître.

MiRANDA-PÉREZ Fabiola \& Gomes-Medina Angélica (2014), « Quelle reconnaissance des droits reproductifs au Chili et en Colombie? », A. Gautier \& C. Grenier-Torres (dir.), Les droits reproductifs 20 ans après Le Caire, Autrepart, 70, 23-40. 
OlEA MAUlEón Cecilia (1998), Encuentros y (des)encuentros busquedas: el movimiento feminista en Amé rica Latina, Lima : Flora Tristan.

RousSEAu Stéphanie (2009), Women's Citizenship in Peru, New York : Palgrave Macmillan.

RozÉE Virginie (2011), « La Communauté internationale et les droits des femmes », M.-

P. Arrizabalaga, D. Burgos-Vigna \& M. Yusta (dir.), Femmes sans frontières. Stratégies transnationales face à la mondialisation, XVIII ${ }^{e}$-XXI $e^{e}$ siècles, Bruxelles : Peter Lang, 193-211.

VerschuUr Christine \& Destremau Blandine (2012), « Féminismes décoloniaux, genre et

développement. Histoire et récits des mouvements de femmes et des féminismes aux Suds », Revue Tiers Monde, 209, 7-18.

\section{NOTES}

1. La notion d'espace de la cause des femmes (Bereni, 2015) est définie comme « la configuration des sites de mobilisation au nom des femmes et pour les femmes dans la pluralité des sphères sociales ». Cette notion met l'accent sur la variété des pôles sectoriels (associatif, partisan, étatique, académique) et des mouvances idéologiques (continuum des positions entre le militantisme féminin et féministe), et met en lumière ce que cette structuration de l'espace de la cause des femmes produit comme forces centrifuges et comme mécanismes de convergences. Définie pour le cas français, cette notion peut à mon sens être élargie à l'espace international, considérant le système onusien et ses agences régionales telle la Commission économique et sociale pour l'Amérique latine (CEPAL).

2. Le Lobby européen des Femmes est créé en 1990, quinze ans après l'adoption de la première directive sur l'égalité de rémunération. L'objectif de cette première structure de mobilisation des femmes au niveau européen est de promouvoir la réalisation de l'égalité des droits et des chances entre les femmes et les hommes par l'action collective institutionnalisée.

3. On peut citer parmi les réseaux thématiques transnationaux latino-américains la Red de Salud de las Mujeres Latinoamericanas y Caribeñas (RSMLAC) ou le Comité Latinoaméricano para la Defensa de los Derechos de las Mujeres (CLADEM) : pour plus de détails, voir Marques-Pereira (2010).

\section{RÉSUMÉS}

L'objet de cette contribution est d'aborder quelques modalités majeures des résistances féministes concernant des enjeux fondamentaux liés à la citoyenneté des femmes. Nous mettrons en lumière que les résistances féministes se sont largement fondées sur la désobéissance à l'ordre des sexes. Désobéir aux normes de l'ordre des sexes renvoie à la transgression de celles-ci, à leur contournement, au détournement de leur sens androcentré ou patriarcal. L'objectif poursuivi est d'illustrer cette désobéissance par quelques exemples européens (Belgique, France, GrandeBretagne) et latino-américains (Cône sud) ayant trait au suffragisme et à la politisation du privé et du corporel, des phénomènes essentiels aux revendications des trois vagues du féminisme : le droit de vote pour la première, le droit à la libre disposition de soi pour la deuxième, et le droit à l'autonomie qui se prolongera lors de la troisième vague du féminisme. Notre démarche s'inscrit dans une perspective socio-historique et politique et comporte deux versants : le premier axé sur 
les processus de subjectivation et d'individuation des femmes qui sont au cœur de leur citoyenneté, le second centré sur les dynamiques institutionnelles et protestataires qui se développent à l'aune de la transnationalisation du féminisme, moteur de cette subjectivation et individuation.

The object of this paper is to consider some major modalities of feminist resistance concerning important issues for women's citizenship. We will show that feminist resistance has been largely based on disobedience to the sex order. To disobey sex order norms means transgressing, avoiding them, detouring their androcentric or patriarchal meaning. Our purpose is to illustrate that disobedience in a few examples from Europe (Belgium, France, UK) and Latin America (South Cone) related to suffragism and to politicization of the private and the body, major phenomena of the three waves of feminism: the right to vote for the first, the right to control one's body and person for the second and the right to autonomy, which will also be claimed during the third feminist wave. Our prospect rests in a socio-historic and political perspective and has two aspects: the first concerns the processes of women's subjectivation and individualization that are at the hart of their citizenship, the second is centred on the institutional and protest dynamics developing in the wake of the transnationalization of feminism driving subjectivation and individualization.

\section{INDEX}

Mots-clés : citoyenneté, désobéissance, subjectivation, individuation, transnationalisation, féminisme

Keywords : citizenship, disobedience, subjectivation, individualization, transnationalization, feminism

\section{AUTEUR}

\section{BÉRENGÈRE MARQUES-PEREIRA}

Bérengère Marques-Pereira est professeure de l'Université libre de Bruxelles (ULB) en sciences politiques et sociales. Elle a été présidente de l'Association belge de science politique (ABSP) et est actuellement co-présidente du groupe « Genre et politique » de l'ABSP qu'elle a créé lors de sa présidence. Ses publications portent sur la citoyenneté sociale et politique des femmes en Europe et en Amérique latine.

bmarques@ulb.ac.be 